\title{
Increased Muscle Sympathetic Nerve Activity during Delayed Auditory Feedback in Humans
}

\author{
Toshiyoshi Matsukawa, Yoshiki Sugryama, \\ and Tadaaki MANO \\ Department of Autonomic and Behavioral Neurosciences, Division of \\ Higher Nervous Control, Research Institute of Environmental \\ Medicine, Nagoya University, Nagoya, 464-01 Japan
}

\begin{abstract}
Summary We examined the effect of mental stress on muscle sympathetic nerve activity (MSNA) by microneurographically measuring the MSNA of the tibial nerve while subjects read aloud. A delayed auditory feedback task (DAF) was employed with a delay interval of $200 \mathrm{~ms}$ and these performances were compared to those in the absence of DAF in five healthy men ( $28 \pm 2$ years). The mean blood pressure was significantly elevated during reading both in the presence of DAF and in its absence, and the pressor response was more exaggerated in the presence of DAF than its absence. MSNA and plasma norepinephrine levels were significantly increased during reading in the presence of DAF but not in its absence, whereas heart rate was mildly increased at the same extent during reading in both the presence and absence of DAF. The results suggest that mental stress produces elevation in blood pressure and mediates the activation of sympathetic nerve activity.
\end{abstract}

Key words : delayed auditory feedback, humans, mental stress, microneurography, muscle sympathetic nerve activity.

Mental stress has been known, by measuring forearm or calf blood flow which reflects mainly muscle blood flow, to induce decreases in vascular resistance in healthy humans, and the decreases in vascular resistance during mental stress have been suggested to be due to inhibition of sympathetic nerve activity [1,2]. However, the results of previous studies regarding the effect of mental stress on directly recorded muscle sympathetic nerve activity (MSNA) in humans is controversial

Received on November 11, 1994; Accepted on August 5, 1995

Correspondence should be addressed to: Toshiyoshi Matsukawa, Department of Autonomic and Behavioral Neurosciences, Division of Higher Nervous Control, Research Institute of Environmental Medicine, Nagoya University, Furo-cho, Chikusa-ku, Nagoya, 464-01 Japan. Tel: +81-52-7893883, Fax: +81-52-789-3887, E-mail: matsu@riem.nagoya-u.ac.jp 
[3-6]. That is, some investigations have shown either no change or slight decreases in MSNA during mental stress [3,4] whereas others have revealed increases in MSNA [5,6]. In this study, a delayed auditory feedback task (DAF) [7,8] was employed as a mental stress task and the effects of DAF on MSNA were examined in normal humans. Usually, we read aloud while hearing our own voices through the both bone and air conduction (auditory feedback). The cognition of our own voices by the auditory feedback during the reading helps us to read aloud fluently. As a DAF mental stress test, subjects are made to read a book aloud while hearing their own voices with a delay interval of $200 \mathrm{~ms}$ through the air conduction via headphones. Therefore, during the reading with the DAF, subjects simultaneously hear their own voices both delayed through the air conduction via headphones as well as with no delay through bone conduction; accordingly, cognitive conflict occurs. The cognitive conflict during reading with the DAF can induce deleterious effects on reading, decreases in fluency and induction errors, and it is hard for subjects to read aloud. Moreover, subjects were also asked to read aloud as rapidly and accurately as possible. Thus, they made efforts to cope with the task, and thereby a certain amount of mental stress can be induced.

We evaluated five healthy men, aged 22 to 34 years (average age $28 \pm 2$ years). They had no hypertension or concurrent illness and received no medication including anti-inflammatory drugs for at least 2 weeks before the study. Written informed consent was obtained from each subject following a detailed explanation of the purpose of the study, the procedures, and possible complications. The protocols had been approved by the Ethical Committee on Human Research of the Research Institute of Environmental Medicine, Nagoya University.

Multi-unit recording of MSNA was obtained from a muscle fascicle of the tibial nerve at the popliteal fossa, and the recorded nerve activity was passed through an integrator with a time constant of $0.1 \mathrm{~s}$ to obtain the mean voltage neurogram of MSNA, according to previous studies [4,9-11]. The spikes were identified as MSNA according to the criteria defined in previous studies [4, 9-11], and the quantitative analysis of MSNA was performed, as follows. Previous reports have shown that the discharge of MSNA was rhythmically synchronous with the heart beat, and that MSNA was discharged with almost constant latencies when triggered by $\mathrm{R}$-waves in ECG [9]. Therefore, the mean voltage neurogram of MSNA was displayed together with the ECG on a pen recorder, and the amount of nerve activity was determined from the tracing. Sympathetic bursts, identified by inspecting the mean voltage neurogram, were expressed as bursts per min. According to the previous studies [9-11], the amplitude of each burst was determined and the integrated total activity was expressed in arbitrary units which were calculated as the product of the burst rate and the mean burst amplitude. The integrated total activity was used to calculate the responses of MSNA to the mental stress task.

After the subject had rested supine for at least $30 \mathrm{~min}$, resting values of mean blood pressure, heart rate, and MSNA were obtained for $10 \mathrm{~min}$. Mean blood pressure was measured intermittently every min using an automatic sphygmomano- 
meter (Nihon Colin, BP-203NP), and heart rate was continuously monitored by electrocardiogram. To determine the resting levels of plasma norepinephrine, blood samples were obtained from the right antecubital vein, which had been cannulated before the study.

Figure 1 shows the block diagram and protocol of this study. A DAF mental stress test was conducted in which subjects read a book aloud for $10 \mathrm{~min}$ while
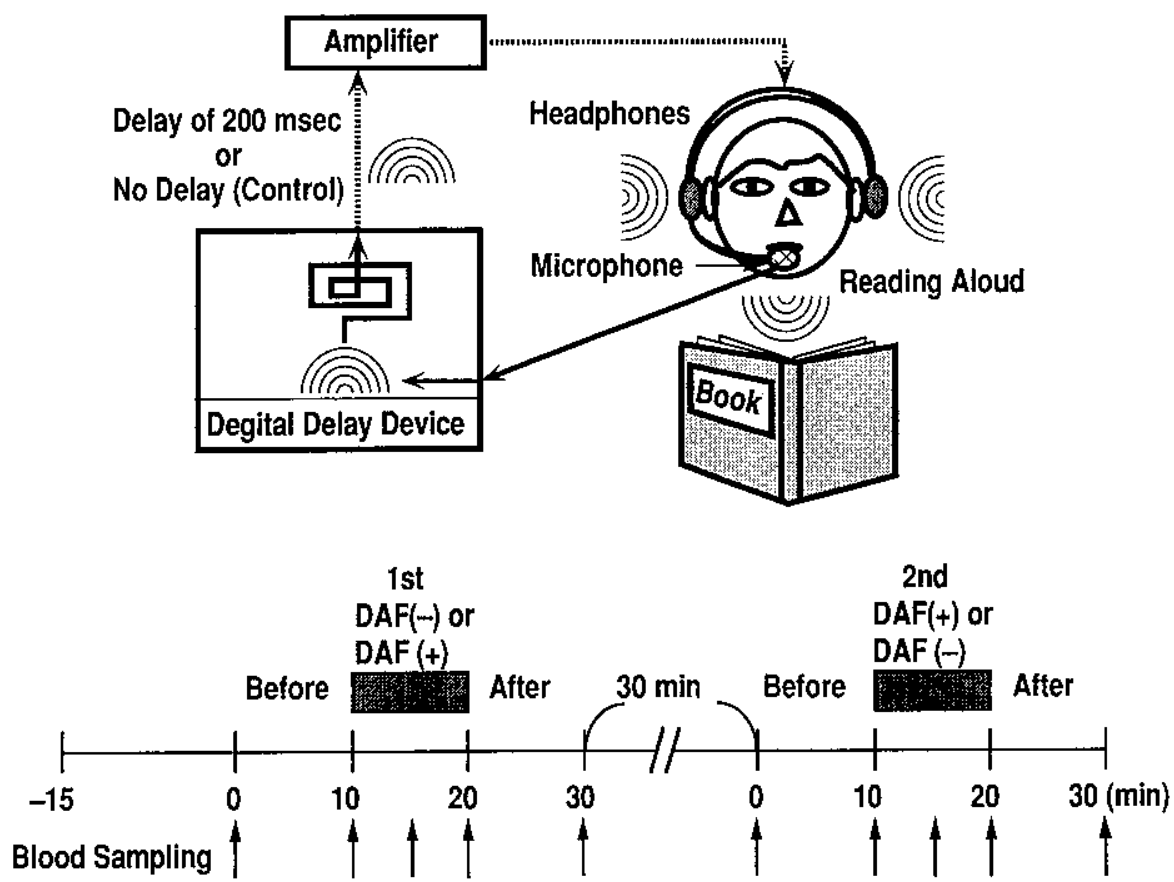

Fig. 1. The block diagram and protocol of this study. The delayed auditory feedback task (DAF) was conducted in which each subject reads aloud for $10 \mathrm{~min}$ from a book while hearing their own voices at a delay of $200 \mathrm{~ms}$ using a digital delay device through headphones. Such a delay in hearing one's voice can have deleterious effects on reading, subjects were asked to read the book as rapidly and as accurately as possible. As a control, each subject was also made to read while hearing their own voices in the absence of delay for $10 \mathrm{~min}$. The delay or no delay audio channels of the instrumentation system were adjusted to provide a unity $(0 \mathrm{~dB})$ acoustic gain from microphone to headphones. Each subject underwent these two tests which were performed according to a randomized design (DAF 50\% vs. its absence 50\%). Mean blood pressure, heart rate, and MSNA were measured before, during, and after reading in the presence and absence of DAF. Blood samples were obtained for measurement of plasma norepinephrine levels from the antecubital vein at $10 \mathrm{~min}$ before, just before reading, at 5 and $10 \mathrm{~min}$ during reading, and at $10 \mathrm{~min}$ after reading during both reading. 
hearing their own voices through headphones with a delay of $200 \mathrm{~ms}$ using a digital delay device (Roland, SDE-3000A) after the voices had been fed into a microphone attached in front of the subjects' mouth. Subjects were also asked to read aloud as rapidly and accurately as possible. As a control study, subjects were also made to read while hearing their own voices with no delay for $10 \mathrm{~min}$. Each subject underwent these two tests which were performed to a randomized design (DAF $50 \%$ vs. its absence $50 \%$ ). The delay or no delay audio channels of the instrumentation system were adjusted to provide a unity $(0 \mathrm{~dB})$ acoustic gain from the microphone to the headphones.

We obtained the averaged values of mean blood pressure, heart rate, and MSNA every $2 \mathrm{~min}$ before, during, and after reading in the presence and absence of DAF. Blood samples were obtained for measurement of plasma norepinephrine levels from the venous cannulae $10 \mathrm{~min}$ before, just before reading, 5 and $10 \mathrm{~min}$ during reading, and $10 \mathrm{~min}$ after reading. Blood samples were collected in chilled, EDTA-2Na tubes and promptly centrifuged. Plasma norepinephrine levels were measured by high-performance liquid chromatography with an electrochemical detector.

Statistical significance was assessed by using analysis of variance and paired or unpaired Student' $t$-test. A probability level of 0.05 was used for statistical significance. Data are presented as the means \pm SE.

An original tracing in one subject and a summary of the results of changes in mean blood pressure, heart rate, MSNA, and plasma norepinephrine levels before,

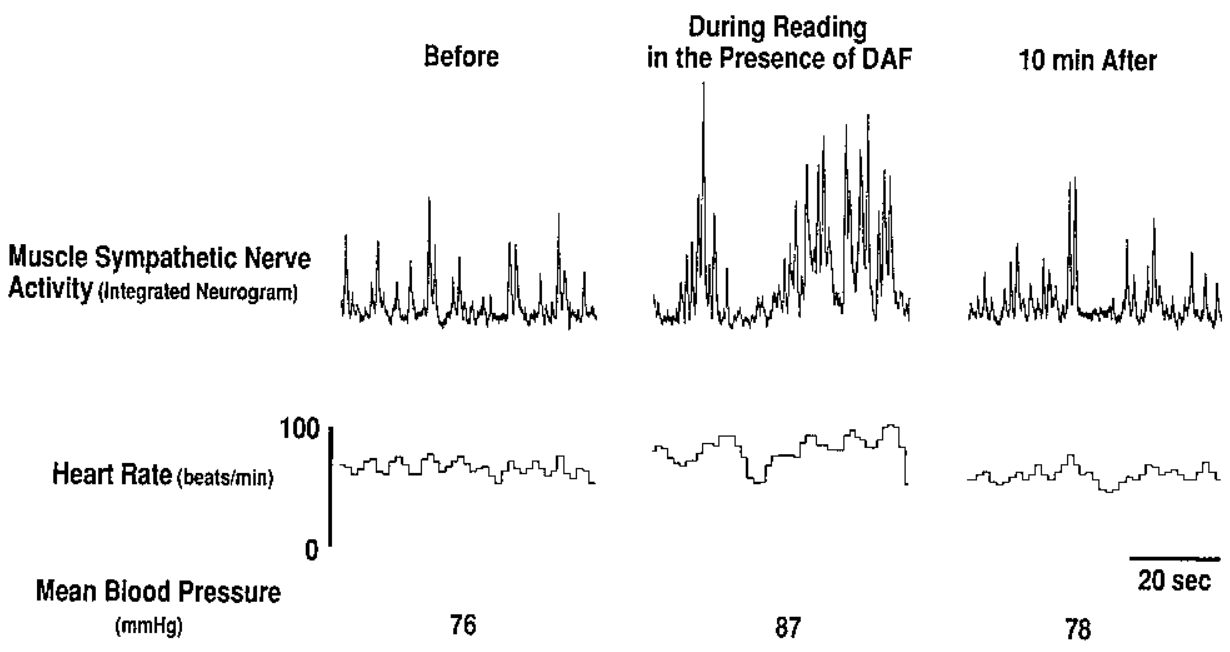

Fig. 2. Original recordings of muscle sympathetic nerve activity, heart rate, and mean blood pressure before, during, and after reading in the presence of delayed auditory feedback (DAF). Each peak represents a spontaneous burst of sympathetic discharge. The frequency and amplitude of sympathetic bursts were elevated during reading in the presence of DAF. 
during, and after reading both in the presence and absence of DAF are shown in Figs. 2 and 3. Mean blood pressure was elevated during reading both in the presence of DAF and in its absence, and the pressor response was significantly greater in the presence of DAF than in its absence $(p<0.05)$ (Fig. 3). The heart rate was increased during reading both in the presence of DAF and in its absence at similar rates during both readings (Fig. 3). MSNA and plasma norepinephrine levels were significantly increased during reading in the presence of DAF but not in its absence $(p<0.05)$ (Fig. 3). Moreover, there was a significant positive correlation between changes in MSNA and those in mean blood pressure during reading in the presence of DAF $(y=0.0232 x+7.489, r=0.694, p<0.05)$ but not in its absence $(y=-0.0319 x+4.541, r=-0.319$, NS).

In the present study, there were some key observations. First, the pressor response in mean blood pressure was enhanced during reading in the presence of DAF as compared with in its absence. Second, MSNA and plasma norepinephrine levels were increased in the presence of DAF but not in its absence, whereas tachycardiac response in the presence of DAF was similar to that in its absence.
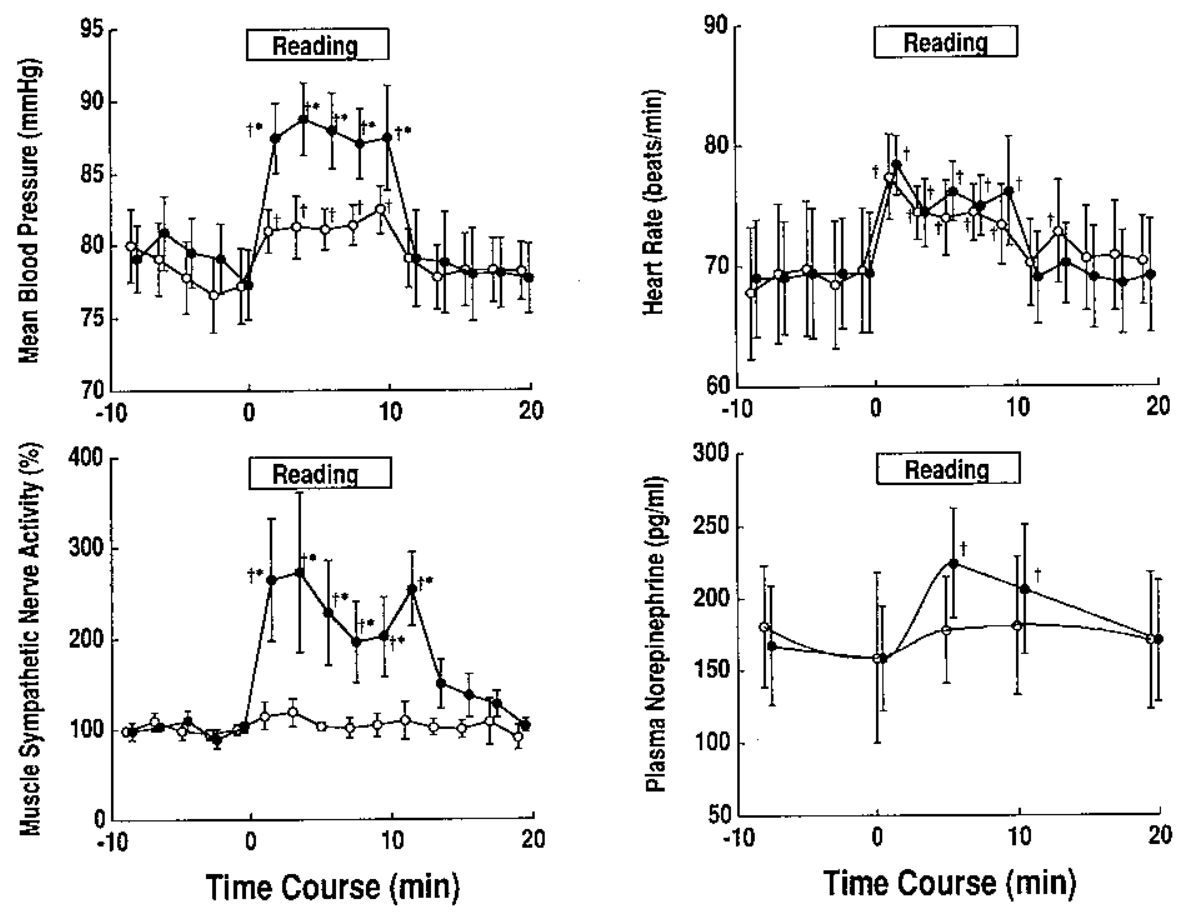

Fig. 3. Mean blood pressure, heart rate, muscle sympathetic nerve activity, and plasma norepinephrine levels before, during, and after reading in the presence of delayed auditory feedback (DAF) (closed circles) and in the absence of DAF (open circles). Data are expressed as means \pm SE. ${ }^{\dagger} p<0.05$ vs. before reading. $*_{p}<0.05$ vs. reading in the absence of DAF. 
Third, there was a significant positive correlation between increases in MSNA and elevations in mean blood pressure in the presence of DAF but not in its absence. MSNA and plasma norepinephrine levels were increased during reading in the presence of DAF but not in its absence, while the tachycardiac response in the presence of DAF was similar to that in its absence. Moreover, there was a significant positive correlation between increases in MSNA and elevations in mean blood pressure in the presence of DAF but not in its absence. Thus, the pressor response observed with DAF seems to be caused by an increase in sympathetic nerve activity. The results lead us to conclude that DAF as mental stress activates the sympathetic nervous system, thereby inducing blood pressure elevation.

Previous reports on the effect of mental stress on MSNA have shown variable results. Short-term (1-2 min) mental stress was usually found to induce either no change or slight decreases in MSNA [3,4], but longer stress periods induce increases in MSNA [5,6]. However, we observed in our present study that increases in MSNA were induced immediately after the initiation of DAF and that these increases continued during the task. The reasons for the difference between the previous results and our results are unclear, but have recently been suggested by Callister and co-workers [12], who demonstrated that MSNA is influenced by the perceptions of stress and the levels of task difficulty during mental stress testing. Namely, the difference in the type of mental stress tasks (mental arithmetic test or color word test in previous studies vs. DAF in this study) [3-6], may contribute to such differences. Thus, the appearance of mental stress as a result of DAF induces increases in sympathetic nerve activity with attendant elevations in blood pressure.

We thank Ms. Hatsue Suzuki for technical assistance and Ms. Fusako Hattori and Ms. Keiko Katou for secretarial support.

\section{REFERENCES}

1. Folkow B: Physiological aspects of primary hypertension. Physiol Rev 62: 347-504, 1982

2. Hjemdahl P, Freyschuss U, Juhlin-Dannfelt A, and Linde A: Differentiated sympathetic activation during mental stress evoked by the Stroop test. Acta Physiol Scand 527 (Suppl): 25-29, 1984

3. Delius W, Hagbarth K-E, Hongell A, and Wallin BG: Manoeuvres affecting sympathetic outflow in human muscle nerves. Acta Physiol Scand 84: 82-94, 1972

4. Matsukawa T, Gotoh E, Uneda S, Miyajima E, Shionoiri H, Tochikubo O, and Ishii, M: Augmented sympathetic nerve activity in response to stressors in young borderline hypertensive men. Acta Physiol Scand 141: 157-165, 1991

5. Hjemdahl P, Fagius J, Freyschuss U, Wallin BG, Daleskog M, Bohlin G, and Perski A: Muscle sympathetic activity and norepinephrine release during mental challenge in humans. Am J Physiol 261: E654-E664, 1989

6. Wallin BG, Esler M, Dorward P, Eisenhofer G, Ferrier C, Westerman R, and Jennings G: Simultaneous measurements of cardiac noradrenaline spillover and sympathetic 
outflow to skeletal muscle in humans. J Physiol (Lond) 453: 45-58, 1992

7. Haywood HC: Differential effects of delayed auditory feedback on palmar sweating, heart rate, pulse pressure. J Speech Hear Res 6: 181-186, 1963

8. Fillenbaum S: Delayed auditory feedback with different delay times at each ear. J Speech Hear Res 7: 369-371, 1964

9. Wallin BG: Intraneural recording and autonomic function in man. In: Autonomic Failure, ed. Banister R, Oxford Univ Press, London, pp 36-51, 1983

10. Matsukawa $T$, Gotoh E, Minamisawa K, Kihara M, Ueda S, Shionoiri H, and Ishii M: Effects of intravenous infusions of angiotensin II on muscle sympäthetic nerve activity in humans. Am J Physiol 261: R690-R696, 1991

11. Matsukawa T, Mano T, Gotoh E, and Ishii M: Elevated sympathetic nerve activity in patients with accelerated essential hypertension. J Clin Invest 92: 25-28, 1993

12. Callister R, Suwarno NO, and Seals DR: Sympathetic activity is inffuenced by task difficulty and stress perception during mental challenge in humans. J Physiol (Lond) 454: 373-387, 1992 